\title{
Effects of generative processes on probe identification time
}

\author{
JOHN G. SEAMON \\ Wesleyan University, Middletown, Connecticut 06457
}

\begin{abstract}
Studies of character classification have shown that reaction time is reduced when the probe is the same as the last-rehearsed target item. If rehearsal functions as a generative process to reduce response time by stimulus activation, comparable results should be obtained if the task is changed from probe classification to probe naming. A letter identification baseline was obtained in Session 1 where subjects named single probe letters as quickly as possible. In Sessions 2 and 3, each probe letter was preceded by a target of three to five letters, which were rehearsed individually prior to the probe, and subjects reported their last rehearsal after naming the probe. The results showed that (1) naming latencies were longer in Sessions 2 and 3 than in Session 1, suggesting that rehearsal requires conscious attention; (2) letter probes that matched the last-rehearsed target item were named faster than those that were different; (3) when performance was examined in terms of the rehearsal distance between the target items and probe, target set size had no effect on probe identification time. Generative processes were suggested to influence probe encoding time through stimulus preprocessing.
\end{abstract}

Character classification time has been hypothesized to reflect the sum of the component times for probe encoding, memory comparison, and response organization and execution (Sternberg, 1969). Recent evidence has suggested that the duration of the probe encoding stage may be reduced by the appropriate use of a generative processing mechanism (Seamon, 1976; Seamon \& Wright, 1976). In these studies subjects were presented with a target array of letters followed by a probe letter a few seconds later. A subject rehearsed each target letter at a self-paced or externally paced rate until the probe arrived, decided if the probe was a member of the preceding target by making a manual response, and reported the last item rehearsed prior to the presentation of the probe. When decision latencies for positive responses were examined as a function of $D$, the computed circular distance between the probe and the last-rehearsed target item, it was found that reaction time increased linearly over target set size at a comparable rate for all values of $D$. Reaction time was significantly faster when the probe was the same as the lastrehearsed item $(D=0)$ than when different $(D>0)$; if different, it did not matter whether the distance between the probe and the last-rehearsed item was one or more character spaces. It was suggested that rehearsal functions as a generative process in which subjects generate representations of possible probes and reduce reaction time through some means of stimulus priming or activation.

This research was supported by NIH Grant 1-RO3-MH 27126-01 awarded to the author. Appreciation is expressed to Dominic Massaro, who suggested the vocal identification test, Catharine Barclay, who assisted in data collection, and the two reviewers, who made helpful suggestions. Requests for reprints should be sent to John G. Seamon, Department of Psychology, Wesleyan University, Middletown, Connecticut 06457.
While the above results are not inconsistent with a response bias explanation, an interpretation emphasizing facilitation of probe encoding by stimulus code activation is favored by the results of studies of stimulus probability and expectancy. These studies have shown that neither stimulus probability (Klatzky \& Smith, 1972) nor expectancy (Shiffrin \& Schneider, 1974) interacts with target set size, but stimulus probability does interact with stimulus quality (Miller \& Pachella, 1973). Target set size affects memory comparison time, while stimulus quality influences only probe encoding time (Sternberg, 1967). It might be maintained, as Biederman and Stacy (1974) have argued, that stimulus quality should interact with target set size if stimulus probability is confounded with set size, as was done by Sternberg (1967). However, the results of Miller and Pachella (1973) indicate that a Stimulus Probability by Quality interaction should be found only if very low probabilities are used. While Sternberg (1967) varied stimulus probability and quality, he did so over a range where little interaction might be expected.

If the effects of stimulus probability and expectancy are comparable (cf. Hinrichs \& Craft, 1971a) and generative processes are viewed in this fashion, a test of the hypothesis that generative processes affect probe encoding time is provided by a probe identification task. Changing the response in a character classification task from probe classification to probe identification should not alter the basic outcome: Probe naming should be faster when the last-rehearsed item matches the probe than when it does not. This finding would indicate that generative processes affect probe encoding time more than response time because (1) stimulus probability and quality interact in probe classification and probe naming in the same fashion (Miller \& Pachella, 1973), and (2) expectancy effects on reaction time are due to cor- 
rect stimulus anticipation and not to response preparation when response difficulty is minimized (Hinrichs \& Craft, 1971b; Hinrichs \& Krainz, 1970).

A second purpose of this research is to determine if target set size influences probe identification time when the effects of generative processes are equated. Kirsner (1972) used the Sternberg classification paradigm, but required probe naming instead of probe classification. Naming latency was faster for probes that were present in the target than for those that were not present, and the facilitation effect decreased as target set size in creased from one to four items. Kirsner argued that, since a memory comparison stage was logically absent in this task, target set size must have an effect upon probe encoding time.

An alternative hypothesis is that Kirsner found no effect of target set size on probe encoding time, but a stimulus repetition effect which is generally confined to the last item in the target when the target-probe interval is short and subjects are instructed not to rehearse the items (Kirsner, 1972). Reaction time has been shown to be faster for repeated than nonrepeated characters in stimulus naming (Eichelman, 1970) and classification (Posner \& Boies, 1971) tasks, and these effects have been attributed to encoding time differences. Essentially the same result may be seen in Kirsner's analysis of reaction time by item position within a target. Naming was facilitated when the probe was the same as the last item in the sequential target array (Kirsner, 1972, p. 174). In terms of the present analysis of generative processes and reaction time, the percentage of characters that a subject would be prepared for under these conditions would show a progressive decline from $100 \%$ in Target Size 1 to $25 \%$ in Target Size 4 . The effect of target set size on probe naming may reflect nothing more than this confounding.

An unbiased test of a target set size effect on probe encoding is provided by examining probe identification time across different set sizes when the rehearsal distance between the target items and the probe has been equated. Since earlier work has not shown generative processes to interact with target set size (Seamon, 1976; Seamon \& Wright, 1976), set size should have no effect on probe identification time.

\section{METHOD}

\section{Subjects}

The subjects included three Wesleyan University students and the experimenter. All subjects were familiar with the character classification paradigm, having participated in at least one previous reaction time study, although none had previous practice with vocal responses.

\section{Apparatus}

All target and probe characters were presented on a rearprojection screen by a Gerbrands projection tachistoscope. Vocal reaction times were obtained from a Durgin and Brown millisecond timer which was activated with the opening of the probe shutter on each trial and terminated by an oral response made into a microphone worn by the subject as part of a headphone set. The subject was seated at a desk and shielded from the tachistoscope and timers by a wall partition.

\section{Procedure}

The experiment was conducted over three sessions, with 180 trials presented in each session. The first session was devoted to obtaining a baseline level of performance in simple letter identification. Subjects were presented with an uppercase English letter in the center of the display screen and the time required to vocalize the name of the letter was recorded. All of the letters of the alphabet were employed, although the multisyllable $W$ was used only once in each session. A trial consisted of an auditory click, followed $2 \mathrm{sec}$ later by a second auditory click (the opening and closing of the shutter on the unused projector), a 3.5-sec delay, and the visual probe letter for $2 \mathrm{sec}$. The reaction times were recorded during a $3-\mathrm{sec}$ intertrial interval.

The same set of probe characters was presented in Sessions 2 and 3 . In these sessions, however, each probe letter was preceded by a visual target of three to five uppercase letters presented simultaneously in a horizontal array. The temporal parameters for a trial were the same as those of Session 1, but the target characters were displayed for $2 \mathrm{sec}$ between two auditory clicks which preceded the probe.

Within each of the last two sessions, the subjects were presented with 60 trials for each random letter string of three, four, and five letters. The probe letter was present in the target on half of the trials for each target set size condition; its position within the target was randomized and approximately equivalent across all positions. Target set size, presence of the probe in the target, and the position of the probe in the target for positive instances were randomized from trial to trial.

As in Session 1, the subjects were instructed to vocally identify the probe character as quickly and as correctly as possible. However, in Sessions 2 and 3 the subjects had an additional requirement of subvocally rehearsing the three to five target characters at a cyclic self-paced rate and vocally identifying the last-rehearsed target letter. The subject thus made two vocalized responses: The first, which triggered the voice key, was an identification of the probe letter; the second was a report of the last letter in the target sequence rehearsed before the appearance of the probe.

\section{RESULTS}

Mean reaction times, standard errors, and number of observations for vocal identifications in Session 1 and vocal identifications and last-rehearsed responses in Sessions 2 and 3 are shown in Table 1 . In each instance the data represent the pooled mean reaction time, standard error, and number of observations for the four

Table 1

Vocal Probe Identification Time for No-Target and Rehearsed Target Trial Condition

\begin{tabular}{|c|c|c|c|c|}
\hline & \multirow{2}{*}{$\frac{\begin{array}{c}\text { Session } 1 \\
\text { (Only Probes) }\end{array}}{\text { All RTs }}$} & \multicolumn{3}{|c|}{$\begin{array}{c}\text { Sessions } 2 \text { and } 3 \\
\text { (Targets Preceded Probes) }\end{array}$} \\
\hline & & Yes $/ D=0$ & Yes $/ D>0$ & No \\
\hline RT & 452 & 524 & 557 & 560 \\
\hline $\mathrm{SE}$ & 4 & 11 & 8 & 6 \\
\hline $\mathrm{N}$ & 173 & 44 & 116 & 168 \\
\hline
\end{tabular}

Note-The data in each row are means pooled over the individual performance of each subject. 
subjects. Trials were lost or excluded for mechanical failures $(3.61 \%$ and $5,21 \%$ ), inappropriate responses $(0 \%$ and $.42 \%$ ), and latencies greater than $900 \mathrm{msec}$ (.28\% and 3.19\%) in Session 1 and Sessions 2 and 3, respectively. For Sessions 2 and 3 the means were calculated separately for probe letters that were (1) present in the target and the same as the last-rehearsed item (yes/D =0), (2) present in the target but different from the last-rehearsed item (yes/D >0), and (3) not present in the target (no).

The results of Sessions 2 and 3 show that vocal reaction times for probe identification are faster when the probe is the same as the last-rehearsed letter than when it differs. The reliability of the $33-\mathrm{msec}$ difference between the yes $/ D=0$ and yes/D $>0$ mean reaction times is supported by the results of a one-tailed test which found the difference in the pooled means to be significant $[t(158)=2.25, p<.02]$ and by the fact that all four of the subjects showed the same pattern of results. This indicates that reaction time is influenced in a vocal identification task in much the same manner as in a same-different classification task. When the probe letter is the same as the last letter the subject has rehearsed from the target sequence $(D=0)$, the reaction time is faster than if the two characters are different $(D>0)$.

Of additional interest in Table 1 is a comparison of the mean reaction time of Session 1 with those of Sessions 2 and 3. Naming a probe letter with no other demands (Session 1) is reliably faster than naming a letter which is preceded by a target array that must be rehearsed and the last rehearsal reported (Sessions 2 and 3 ). These results are consistent with the observations of Posner and Boies (1971) and Posner and Klein (1973), who indicate that rehearsal requires conscious attention. While the processing of a single letter may be described as a process of effortless retrieval (Posner \& Klein, 1973), the probe latencies for Sessions 2 and 3 may be lengthened, as the central processor must then allocate some of its limited capacity to rehearsing the target characters, vocalizing the probe name after it has been processed, and, lastly, remembering and recalling the last letter rehearsed. Less processing capacity would appear to be left for vocalizing the probe name in Sessions 2 and 3 than in Session 1.

The effects of target set size upon probe identification are shown in Table 2, which presents reaction times for probe letters in Sessions 2 and 3. As before, each observation represents the mean score based on the pooled performance of the four subjects. Mean reaction times do not increase over target set size for probes that were present in the target (yes $/ D=0$ or $D>0$ ) or not present in the target (no). This finding indicates that the number of characters in the target array does not influence the probe encoding process and is consistent with Sternberg (1967), who has indicated that target set size affects only the memory comparison stage, a
Table 2

Probe Identification Time for Each Target Set Size and Response Condition for Sessions 2 and 3

\begin{tabular}{|c|c|c|c|c|}
\hline \multirow[b]{2}{*}{ Condition } & & \multicolumn{3}{|c|}{ Target Set Size } \\
\hline & & 3 & 4 & 5 \\
\hline No & $\begin{array}{l}\text { RT } \\
\text { SE } \\
N\end{array}$ & $\begin{array}{r}558 \\
11 \\
55\end{array}$ & $\begin{array}{r}558 \\
10 \\
54\end{array}$ & $\begin{array}{r}564 \\
10 \\
59\end{array}$ \\
\hline Yes/D $>0$ & $\begin{array}{l}\text { RT } \\
\text { SE } \\
\mathrm{N}\end{array}$ & $\begin{array}{r}571 \\
16 \\
35\end{array}$ & $\begin{array}{r}537 \\
12 \\
39\end{array}$ & $\begin{array}{r}566 \\
14 \\
41\end{array}$ \\
\hline Yes $/ D=0$ & $\begin{array}{l}\text { RT } \\
\text { SE } \\
N\end{array}$ & $\begin{array}{r}538 \\
16 \\
19\end{array}$ & $\begin{array}{r}507 \\
17 \\
13\end{array}$ & $\begin{array}{r}523 \\
23 \\
12\end{array}$ \\
\hline
\end{tabular}

Note-The data in each row are means pooled over the individual performance of each subject.

processing stage that was eliminated in the present experiment. The present results are in sharp contrast with the results of Kirsner (1972) and provide support for the hypothesis that those findings reflect a confounding of stimulus preparation with target set size.

The mean reaction time for each rehearsal distance function along with their expected and observed percentage of occurrence is presented in Table 3 . Expected percentages change because Distance Functions $D=0,1$, and 2 could be observed in all target set sizes, while $\mathrm{D}=3$ could be found only in Target Sets 4 and 5 , and $D=4$ could occur only in Target Set 5 . No significant difference $\left[\chi^{2}(4)=3.24, p>.50\right]$ was found between the expected and observed frequencies for the five distance functions.

Since latencies did not vary over target set size, these data were collapsed to yield more observations per condition for each subject and then pooled over subjects. No systematic change in reaction time can be seen for the $D=1-4$ functions in Table 3 or in an examination of the data from the four subjects individually. Consistent with the previously cited classification data (Seamon, 1976; Seamon \& Wright, 1976), these results indicate that only the letter currently being rehearsed within a target receives attention and is capable of reducing reaction time. If the probe and the last-rehearsed letter

Table 3

Probe Identification Time and Expected and Observed Percentages of Each Rehearsal Distance Function

\begin{tabular}{lccccc} 
& \multicolumn{5}{c}{ Rehearsal Distance Function } \\
\cline { 2 - 6 } & $\mathrm{D}=0$ & $\mathrm{D}=1$ & $\mathrm{D}=2$ & $\mathrm{D}=3$ & $\mathrm{D}=4$ \\
\hline $\mathrm{RT}$ & 524 & 563 & 558 & 544 & 554 \\
$\mathrm{SE}$ & 11 & 13 & 15 & 18 & 26 \\
$\mathrm{~N}$ & 44 & 39 & 37 & 24 & 10 \\
Expected & $26.11 \%$ & $26.11 \%$ & $26.11 \%$ & $15.00 \%$ & $6.66 \%$ \\
Observed & $27.43 \%$ & $24.45 \%$ & $23.35 \%$ & $14.73 \%$ & $6.11 \%$ \\
\hline
\end{tabular}

Note-Data are based on positive responses. The data in each row were collapsed over all target set sizes and then pooled over individual subjects. 
are different, it does not matter how many other letters the subject has rehearsed since the probe character.

\section{DISCUSSION}

It was shown that effects of generative processes on reaction time found in a character classification task (Seamon, 1976; Seamon \& Wright, 1976) can be observed in a naming task. Letter probes that matched the last-rehearsed target item $(D=0)$ were named faster than those that were different (D $>0$ ), and, if different, the number of character spaces between the probe and the last rehearsal $(D=1-4)$ did not matter. When performance was examined in terms of the rehearsal distance functions, target set size had no effect on probe identification time.

The present data and the previous classification data are consistent with the hypothesis that generative processes influence probe encoding time. While a response bias interpretation cannot be eliminated, the previously cited studies of stimulus probability and expectancy strongly suggest that the present results reflect encoding effects more than response preparation effects. Clearly, generative processes cannot affect a memory comparison process, as this stage was logically absent in the present naming task. It might seem reasonable to determine if generative processes interact with an encoding variable like stimulus quality in either a probe classification or probe naming task. However, if the effects of generative processes and stimulus probability are comparable and a Stimulus Probability by Quality interaction is found only when very small probabilities (2.5\%.7.5\%) are employed (Miller \& Pachella, 1973), the likelihood of finding an interaction is remote, as the $D=0$ probabilities remain relatively high $(100 \%$ 14.28\%) over Target Set Sizes 1-7.

One way of viewing the present results is to assume that generative processes are a form of stimulus preprocessing that affects primarily probe encoding. While conscious attention is not needed to encode a familiar letter (Posner \& Klein, 1973), generative processes can, through activation or priming, influence the perceptual encoding of that letter when it occurs. Rate of processing can be a variable even though this processing may be automatic in the sense of not needing attention (LaBerge \& Samuels, 1974). Rehearsing a character can be thought of as a preprocessing activity which influences probe pattern recognition processes by activating a particular letter's representational code or processing pathway (Posner \& Boies, 1971) prior to external stimulation. Preprocessing the right stimulus may reduce the amount of information needed to be extracted from an external stimulus before its representational code reports that it occurred, while preprocessing the wrong stimulus may produce conflict among the codes and increase the amount of information needed to be extracted. Encoding time would thus be seen to vary directly with the amount of information extraction necessary for pattern recognition.

\section{REFERENCES}

Biederman, I., \& Stacy, E. W., JR. Stimulus probability and stimulus set size in memory scanning. Joumal of Experimental Psychology, 1974, 102, 1100-1107.

Eichelman, W. H. Stimulus and response repetition effects for naming letters at two response-stimulus intervals. Perception \& Psychophysics, 1970, 7, 94.96.

Hinrichs, J. V., \& CRAFT, J. L. Verbal expectancy and probability in two-choice reaction time. Journal of Experimental Psychology, 1971, 88, 367-371. (a)

Hinfichs, J. V., \& Craft, J. L. Stimulus and response factors in discrete choice reaction time. Journal of Experimental Psychology, 1971, 91, 305-309. (b)

Hinrichs, J. V., \& Krainz, P. L. Expectancy in choice reaction time: Anticipation of stimulus or response? Journal of Experimental Psychology, 1970, 85, 330-334.

KIRSNER, K. Naming latency facilitation: An analysis of the encoding component in recognition reaction time. Joumal of Experimental Psychology, 1972, 95, 171-176.

Klatzky, R. L., \& Sмith, E. E. Stimulus expectancy and retrieval from short-term memory. Journal of Experimental Psychology, 1972, 94, 101-107.

LaBerge, D., \& Samuels, S. J. Toward a theory of automatic information processing in reading. Cognitive Psychology, 1974, 6, 293-323.

Miller, J. O., \& Pachella, R. G. Locus of the stimulus probability effect. Joumal of Experimental Psychology, 1973, 101, 227-231.

Posner, M. I.. \& Boies. S. J. Components of attention. Psychological Review, 1971, 78, 391-408.

Posner. M. I., \& Klein, R. On the functions of consciousness. In S. Kornblum (Ed.), Attention and performance IV. New York: Academic Press, 1973.

SEAmon, J. G. Generative processes in character classification: II. A refined testing procedure. Bulletin of the Psychonomic Society. 1976, 7, 327-330.

Seamon, J. G., \& Wright, C. E. Generative processes in character classification: Evidence for a probe encoding set. Memory \& Cognition, 1976, 4. 96-102.

Shiffrin, R. M., \& Schneider, W. An expectancy model for memory search. Memory \& Cognition, 1974, 2, 616-628.

STERnBERG, S. Two operations in character recognition: Some evidence from reaction-time measurements. Perception \& Psychophysics, 1967, 2, 45-53.

STERnBerg, S. Memory-scanning: Mental processes revealed by reaction-time experiments. American Scientist, 1969, 57, 421.457.

(Received for publication March 11, 1976; revision received June 2,1976 .) 